UDC $636.52 / .58 .085 .2$

637.5 ' 65.053

Original research paper

doi: 10.5937/AASer1846247D

Acta Agriculturae Serbica, Vol. XXIII, 46 (2018); 247-255

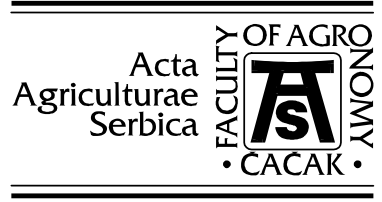

\title{
Carcass conformation of Master Gris broiler chickens as an indicator of carcass quality
}

\author{
Vladimir Dosković ${ }^{1}$, Snežana Bogosavljević-Bošković ${ }^{1}$, Zdenka \\ $\breve{S} k$ rbbić $^{2}$, Boban Jašović ${ }^{3}$, Simeon Rakonjac ${ }^{1}$, Veselin Petričević ${ }^{2}$, \\ Milun D. Petrović ${ }^{1}$
}

${ }^{1}$ Faculty of Agronomy, University of Kragujevac, Cara Dušana 34, 32000 Čačak

${ }^{2}$ Institute for Animal Husbandry, Autoput 16, P. Fah 23, 11080, Belgrade-Zemun

${ }^{3}$ Faculty of Agriculture, University of Pristina, Kosovska Mitrovica-Zubin potokLešak, 38219 Lešak

Corresponding author:vlade321@gmail.com

\begin{abstract}
This study was conducted to evaluate the effect of different feed formulations, fattening period and sex of broiler chickens on absolute carcass conformation measurements (metatarsus length, keel length, breast depth, breast angle and thigh girth) and conformation indices of medium-growing Master Gris chickens. The results showed that, as induced by intensive fattening, body weights were greater and carcass quality, expressed through conformation measurements, was better than the values characteristic of this hybrid (as the hybrid is designed for extensive farming). Regardless of the time of slaughter (at 49 or 63 days of the fattening trial), male chickens had better carcass conformation values $(\mathrm{P}<0.05)$, compared to females, and the 14-day extension of the fattening period led to better values for both absolute and relative carcass conformation measurements in both males and females $(\mathrm{P}<0.05)$.
\end{abstract}

Keywords: chickens, body conformation, diet, broiler age, sex.

\section{Introduction}

Modern chicken meat production is dominated by fast-growing hybrids, characterized by very rapid intensive increases in body weight, high yields of 
first-class meat (breast, thighs and drumsticks), low feed consumption per $1 \mathrm{~kg}$ weight gain, good vitality and resistance. Somewhat lower quality of broiler meat produced under such intensive conditions (watery, tasteless, often too fatty meat), an unstable market price and a high supply of meat are the most likely reasons for the changed consumer opinion that the chicken meat obtained in this way is not so healthy and natural, especially when taking into consideration the fact that all these changes in meat quality and quantity have occurred over a relatively short period of time. At the same time, there have been increasing demands for improved animal welfare (EU), as well as for the prevention of pollution and environmental preservation. This has led to demands for the return to natural sustainable poultry farming using new systems, involving genotype changes and modifications in farming conditions, diet, length of fattening period, etc. (Van Horne et al., 2004, Reddish and Liburn, 2004, Rakonjac 2016, Dosković et al., 2016). On the other hand, there is relatively little data on the scientific justification of new poultry fattening technologies.

Carcass quality can be evaluated using absolute carcass conformation measurements (thigh girth, breast depth, breast angle, Pavlovski et al. 2006) as they are taken on major carcass parts (breast, thighs and drumsticks), as well as using relative carcass conformation measurements (Škrbić et al., 2011), with carcass conformation measurements correlated more strongly with body weight than with genotype (Hopić et al., 2002).

The objective of the present research was to evaluate the quality of meat of male and female medium-growing Master Gris chickens produced during an extended feeding trial (49 i.e. 63 days).

\section{Materials and Methods}

Medium-growing Master Gris chickens were used in a 63-day feeding trial. Standard fattening technology was employed (deep litter, stocking density 10 chickens $/ \mathrm{m}^{2}, 24$ hours of light, ad libitum feeding,...). Fattening involved a threestage feeding programme: starter (0-21 days of age), grower (22-42 days of age) and finisher (43-63 days of age). Chickens were fed diets designed for fastgrowing hybrids (Cobb 500) and were allocated to three groups i.e. treatments with 100 chickens per group (control C - without protease; E-I with $0.2 \%$ protease and E-II with $0.3 \%$ protease supplementation, with crude protein levels reduced by 4 and $6 \%$, respectively, relative to control $\mathrm{C}$ broilers).

\section{Data collection}

At 49 and 63 days of the feeding trial, 30 males and as many females were randomly selected (10 male and 10 female chickens from each treatment), and slaughtered after a fasting period of 8 hours. Carcasses were dissected into primal cuts (according to the Commission Regulation (EC) No 543/2008) and the 
following absolute conformation measurements were taken: breast angle BA (degrees), metatarsus length $\mathrm{ML}(\mathrm{mm})$, keel length $\mathrm{KL}(\mathrm{mm})$, breast depth BD (mm) and thigh girth TG (mm). Using these measurements, based on the correlation between body weight and absolute conformation measurements, relative conformation measurements were calculated - the so-called conformation indices: body weight at slaughter /metatarsus length $(\mathrm{g} / \mathrm{mm})$ BW/ML, body weight at slaughter/keel length $(\mathrm{g} / \mathrm{mm}) \mathrm{BW} / \mathrm{KL}$, body weight at slaughter/breast depth $(\mathrm{g} / \mathrm{mm})$ BW/BD and body weight at slaughter/thigh girth (g/mm) BW/TG).

\section{Statistical analysis}

The obtained data were subjected to statistical analysis using the Statistika (Version 5) software (Stat.Soft.Inc, 2006) by calculating mean values and coefficients of variation ( $\mathrm{Cv}$ ), as well as by testing the significance of differences in the average values of the traits analyzed - using the analysis of variance (for three factors - diet, fattening period and sex of chickens) and Tukey's test for individual comparisons of the tested carcass quality parameters.

\section{Results and Discussion}

Table 1. presents absolute carcass conformation measurements for Master Gris broiler chickens across the tested factors (diet, fattening period, sex).

As indicated in Table 1, the 14-day extension of the fattening period (from 49 to 63 days) led to an increase in all body conformation measurements $(\mathrm{P}<0.05)$, with the smallest difference in breast angle values $(\mathrm{P}>0.05)$. Males had higher values for conformation measurements than females, with only breast angle showing no significance $(\mathrm{P}>0.05)$. The lowest significance was found for the effect of diet, indicating that chickens responded similarly to modifications in complete feeds (feed protein reduction and protease supplementation), regardless of the time of slaughter or sex of broilers (there was no interaction among factors), which was somewhat expected given the complete feed formulation based on protease manufacturer's recommendations (Ronozyme ProAct, DSM, The Netherlands). Master Gris chickens had lower values for carcass conformation measurements compared to fast-growing Cobb 500 birds under similar housing and diet conditions (Dosković et al., 2016), but their values for absolute conformation measurements were better than those of Master Gris chickens at 91 days under free-range system (Blagojević, 2011). 
Table 1. Absolute carcass conformation measurements

\begin{tabular}{|c|c|c|c|c|c|c|c|c|c|}
\hline \multicolumn{3}{|c|}{ Treatment } & & \multirow[b]{2}{*}{$\begin{array}{c}\text { BW, } \\
\text { gr }\end{array}$} & \multirow[b]{2}{*}{$\begin{array}{l}\text { ML, } \\
\mathrm{mm}\end{array}$} & \multirow[b]{2}{*}{$\begin{array}{l}\mathrm{KL}, \\
\mathrm{mm}\end{array}$} & \multirow[b]{2}{*}{$\begin{array}{l}\mathrm{BD}, \\
\mathrm{mm}\end{array}$} & \multirow[b]{2}{*}{$\begin{array}{l}\text { BA, } \\
\text { degrees }\end{array}$} & \multirow[b]{2}{*}{$\begin{array}{l}\text { TG, } \\
\mathrm{mm}\end{array}$} \\
\hline Protease & $\begin{array}{c}\text { Fattening } \\
\text { period, } \\
\text { days }\end{array}$ & Sex & & & & & & & \\
\hline \multirow{8}{*}{ No } & \multirow{4}{*}{49} & \multirow{2}{*}{$\sigma^{\lambda}$} & $\bar{x}$ & $2743.5^{\text {cd }}$ & $82.0^{\mathrm{bc}}$ & $120.8^{c}$ & $103.3^{\text {cd }}$ & 128.6 & $148.7^{\text {cde }}$ \\
\hline & & & $\mathrm{Cv}$ & 2.6 & 2.6 & 2.5 & 4.1 & 1.2 & 3.8 \\
\hline & & \multirow{2}{*}{ q } & $\bar{x}$ & $2397.0^{\mathrm{ef}}$ & $75.7^{d}$ & $113.4^{\mathrm{e}}$ & $99.8^{\text {cd }}$ & 125.6 & $142.6^{\mathrm{e}}$ \\
\hline & & & $\mathrm{Cv}$ & 3.4 & 4.6 & 2.2 & 6.2 & 2.8 & 4.8 \\
\hline & \multirow{4}{*}{63} & \multirow{2}{*}{$\sigma^{\lambda}$} & $\bar{x}$ & $3721.5^{\mathrm{a}}$ & $94.0^{\mathrm{a}}$ & $128.8^{\mathrm{ab}}$ & $113.5^{\mathrm{ab}}$ & 127.9 & $168.5^{\mathrm{a}}$ \\
\hline & & & $\mathrm{Cv}$ & 5.8 & 5.1 & 3.2 & 5.6 & 1.8 & 3.4 \\
\hline & & \multirow{2}{*}{ q } & $\bar{x}$ & $3052.5^{\mathrm{b}}$ & $81.3^{\mathrm{bcd}}$ & $123.8^{\mathrm{bc}}$ & $105.6^{\mathrm{bc}}$ & 127.6 & $152.3^{\text {bcc }}$ \\
\hline & & & $\mathrm{Cv}$ & 7.5 & 3.6 & 5.7 & 5.9 & 2.4 & 4.8 \\
\hline \multirow{8}{*}{$0.2 \%$} & \multirow{4}{*}{49} & \multirow{2}{*}{$\sigma^{\lambda}$} & $\bar{x}$ & $2624.5^{\mathrm{de}}$ & $83.6^{\mathrm{bc}}$ & $121.6^{\mathrm{c}}$ & $105.0^{\mathrm{C}}$ & 128.1 & $154.6^{\mathrm{bc}}$ \\
\hline & & & $\mathrm{Cv}$ & 6.0 & 4.3 & 3.6 & 7.6 & 1.1 & 4.1 \\
\hline & & \multirow{2}{*}{ q } & $\bar{x}$ & $2279.5^{f}$ & $76.1^{d}$ & $114.3^{\mathrm{de}}$ & $95.4^{\mathrm{d}}$ & 128.3 & $144.9^{\text {de }}$ \\
\hline & & & $\mathrm{Cv}$ & 2.1 & 4.8 & 3.5 & 3.5 & 1.3 & 3.0 \\
\hline & \multirow{4}{*}{63} & \multirow{2}{*}{ o } & $\bar{x}$ & $3630.5^{\mathrm{a}}$ & $94.9^{\mathrm{a}}$ & $128.9^{\mathrm{ab}}$ & $113.8^{\mathrm{a}}$ & 127.4 & $164.7^{\mathrm{a}}$ \\
\hline & & & $\mathrm{Cv}$ & 7.1 & 5.1 & 3.0 & 4.6 & 2.6 & 5.0 \\
\hline & & \multirow{2}{*}{ 우 } & $\bar{x}$ & $3037.5^{\mathrm{b}}$ & $82.6^{\mathrm{bc}}$ & $123.3^{\mathrm{bc}}$ & $103.8^{\mathrm{c}}$ & 127.5 & $150.6^{\text {cde }}$ \\
\hline & & & $\mathrm{Cv}$ & 3.8 & 4.7 & 3.4 & 3.6 & 0.9 & 3.0 \\
\hline \multirow{8}{*}{$0.3 \%$} & \multirow{4}{*}{49} & \multirow{2}{*}{ o } & $\bar{x}$ & $2699.5^{\mathrm{d}}$ & $86.2^{\mathrm{b}}$ & $120.2^{\text {cd }}$ & $105.6^{\mathrm{bc}}$ & 127.7 & $148.5^{\text {cde }}$ \\
\hline & & & $\mathrm{Cv}$ & 4.2 & 4.7 & 1.9 & 2.5 & 2.1 & 3.9 \\
\hline & & \multirow{2}{*}{ q } & $\bar{x}$ & $2327.5^{f}$ & $79.7^{\text {cd }}$ & $113.9^{\mathrm{e}}$ & $99.8^{\text {cd }}$ & 126.3 & $141.9^{\mathrm{e}}$ \\
\hline & & & $\mathrm{Cv}$ & 5.9 & 5.3 & 3.6 & 6.3 & 2.2 & 5.91 \\
\hline & \multirow{4}{*}{63} & \multirow{2}{*}{ o } & $\bar{x}$ & $3650.0^{\mathrm{a}}$ & $95.1^{\mathrm{a}}$ & $130.1^{\mathrm{a}}$ & $113.3^{\mathrm{ab}}$ & 129.5 & $160.9^{\mathrm{ab}}$ \\
\hline & & & $\mathrm{Cv}$ & 5.0 & 3.7 & 1.0 & 5.0 & 6.7 & 3.2 \\
\hline & & 0 & $\bar{x}$ & $2955.0^{\mathrm{b}}$ & $82.4^{\mathrm{bc}}$ & $123.0^{\mathrm{bc}}$ & $103.7^{\mathrm{C}}$ & 128.3 & $151.1^{\text {cde }}$ \\
\hline & & 7 & $\mathrm{Cv}$ & 4.2 & 5.0 & 4.3 & 2.5 & 4.9 & 4.1 \\
\hline p-value & & & & & & & & & \\
\hline Source & f variation & & & & & & & & \\
\hline Proteas & & & & 0.039 & 0.042 & 0.936 & 0.579 & 0.815 & 0.076 \\
\hline Fatenin & period & & & 0.000 & 0.000 & 0.000 & 0.000 & 0.392 & 0.000 \\
\hline Sex & & & & 0.000 & 0.000 & 0.000 & 0.000 & 0.184 & 0.000 \\
\hline Proteas & $\mathrm{x}$ fatening & riod & & 0.405 & 0.127 & 0.743 & 0.464 & 0.305 & 0.056 \\
\hline Proteas & $\mathrm{x}$ sex & & & 0.657 & 0.971 & 0.964 & 0.229 & 0.539 & 0.388 \\
\hline Fatenin & period $\mathrm{x}$ se & & & 0.000 & 0.000 & 0.465 & 0.142 & 0.506 & 0.012 \\
\hline Proteas & $\mathrm{x}$ fatening & riod & & 0.830 & 0.879 & 0.659 & 0.662 & 0.669 & 0.431 \\
\hline
\end{tabular}

BW- body weight at slaughter, ML-Metatarsus length, KL-Keel length, BD-Breast depth, BABreast angle, TG-Thigh girth

${ }^{\mathrm{a}-\mathrm{f}}$ Means within a column with different superscripts differ significantly $(\mathrm{P}<0.05)$

In medium-growing RedBro chickens, all body conformation measurements, except metatarsus length, were also significantly greater in chickens slaughtered at day 50 than in birds at 42 days (Pavlovski et al., 2006). 
Acta Agriculturae Serbica, Vol. XXIII, 46(2018); 247-255

Table 2. Carcass conformation indices

\begin{tabular}{|c|c|c|c|c|c|c|c|}
\hline \multicolumn{3}{|c|}{ Treatment } & & \multirow[b]{2}{*}{$\begin{array}{c}\mathrm{BW} / \mathrm{ML}, \\
\mathrm{g} / \mathrm{mm}\end{array}$} & \multirow[b]{2}{*}{$\begin{array}{c}\mathrm{BW} / \mathrm{KL} \text {, } \\
\mathrm{g} / \mathrm{mm}\end{array}$} & \multirow{2}{*}{$\begin{array}{c}\mathrm{BW} / \mathrm{BD}, \\
\mathrm{g} / \mathrm{mm}\end{array}$} & \multirow[b]{2}{*}{$\begin{array}{c}\mathrm{BW} / \mathrm{TG}, \\
\mathrm{g} / \mathrm{mm}\end{array}$} \\
\hline Protease & $\begin{array}{c}\text { Fattening } \\
\text { period, days }\end{array}$ & Sex & & & & & \\
\hline \multirow{8}{*}{ No } & \multirow{4}{*}{49} & \multirow{2}{*}{$\hat{\sigma}$} & $\bar{x}$ & $33.5^{\text {cd }}$ & $22.7^{\text {cd }}$ & $26.6^{\text {cd }}$ & $18.5^{\mathrm{cd}}$ \\
\hline & & & $\mathrm{Cv}$ & 3.5 & 2.8 & 4.8 & 4.6 \\
\hline & & \multirow{2}{*}{ 우 } & $\bar{x}$ & $31.7^{\mathrm{de}}$ & $21.1^{\mathrm{de}}$ & $24.1^{\mathrm{e}}$ & $16.8^{\mathrm{e}}$ \\
\hline & & & $\mathrm{Cv}$ & 6.7 & 4.4 & 6.2 & 5.1 \\
\hline & \multirow{4}{*}{63} & \multirow{2}{*}{$\sigma^{\lambda}$} & $\bar{x}$ & $39.6^{\mathrm{a}}$ & $28.9^{\mathrm{a}}$ & $32.8^{\mathrm{a}}$ & $22.1^{\mathrm{a}}$ \\
\hline & & & $\mathrm{Cv}$ & 6.0 & 5.7 & 5.7 & 3.4 \\
\hline & & \multirow{2}{*}{ 우 } & $\bar{x}$ & $37.5^{\mathrm{ab}}$ & $24.7^{\mathrm{b}}$ & $28.9^{\mathrm{bc}}$ & $20.0^{\mathrm{bc}}$ \\
\hline & & & $\mathrm{Cv}$ & 5.9 & 7.8 & 5.1 & 5.7 \\
\hline \multirow{8}{*}{$0.2 \%$} & \multirow{4}{*}{49} & \multirow{2}{*}{$\sigma^{\pi}$} & $\bar{x}$ & $31.4^{\mathrm{de}}$ & $21.6^{\text {de }}$ & $25.1^{\mathrm{de}}$ & $16.9^{\mathrm{de}}$ \\
\hline & & & $\mathrm{Cv}$ & 5.2 & 7.1 & 9.4 & 4.2 \\
\hline & & \multirow{2}{*}{ 우 } & $\bar{x}$ & $30.0^{\mathrm{e}}$ & $19.9^{\mathrm{e}}$ & $23.9^{\mathrm{e}}$ & $15.7^{\mathrm{e}}$ \\
\hline & & & $\mathrm{Cv}$ & 4.8 & 3.8 & 3.9 & 3.7 \\
\hline & \multirow{4}{*}{63} & \multirow{2}{*}{ 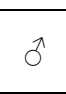 } & $\bar{x}$ & $38.3^{\mathrm{ab}}$ & $28.1^{\mathrm{a}}$ & $31.9^{\mathrm{a}}$ & $22.0^{\mathrm{a}}$ \\
\hline & & & $\mathrm{Cv}$ & 6.6 & 5.5 & 7.1 & 6.0 \\
\hline & & \multirow{2}{*}{ 우 } & $\bar{x}$ & $36.8^{\mathrm{ab}}$ & $24.6^{\mathrm{b}}$ & $29.3^{\mathrm{b}}$ & $20.2^{\mathrm{b}}$ \\
\hline & & & $\mathrm{Cv}$ & 4.1 & 4.5 & 4.9 & 5.1 \\
\hline \multirow{8}{*}{$0.3 \%$} & \multirow{4}{*}{49} & \multirow{2}{*}{ ๙ } & $\bar{x}$ & $31.4^{\mathrm{de}}$ & $22.5^{\mathrm{cd}}$ & $25.6^{\mathrm{de}}$ & $18.2^{\mathrm{de}}$ \\
\hline & & & $\mathrm{Cv}$ & 7.7 & 4.5 & 5.2 & 3.0 \\
\hline & & \multirow{2}{*}{ 우 } & $\bar{x}$ & $29.3^{\mathrm{e}}$ & $20.5^{\mathrm{e}}$ & $23.4^{\mathrm{e}}$ & $16.4^{\mathrm{e}}$ \\
\hline & & & $\mathrm{Cv}$ & 8.0 & 7.0 & 6.2 & 8.7 \\
\hline & \multirow{4}{*}{63} & \multirow{2}{*}{ o } & $\bar{x}$ & $38.4^{\mathrm{ab}}$ & $28.0^{\mathrm{a}}$ & $32.3^{\mathrm{a}}$ & $22.7^{\mathrm{a}}$ \\
\hline & & & $\mathrm{Cv}$ & 5.2 & 4.2 & 6.2 & 4.1 \\
\hline & & \multirow{2}{*}{ 우 } & $\bar{x}$ & $35.9^{b c}$ & $24.0^{\mathrm{bc}}$ & $28.5^{\mathrm{bc}}$ & $19.5^{\mathrm{bc}}$ \\
\hline & & & $\mathrm{Cv}$ & 7.5 & 3.5 & 3.0 & 2.4 \\
\hline \multicolumn{8}{|c|}{ p-value } \\
\hline \multicolumn{8}{|c|}{ Source of variation } \\
\hline Proteas & & & & 0.032 & 0.018 & 0.145 & 0.029 \\
\hline Fatenin & period & & & 0.000 & 0.000 & 0.000 & 0.000 \\
\hline Sex & & & & 0.000 & 0.000 & 0.000 & 0.000 \\
\hline Proteast & $\mathrm{x}$ fatening peri & & & 0.563 & 0.175 & 0.729 & 0.005 \\
\hline Proteas & $\mathrm{x}$ sex & & & 0.660 & 0.732 & 0.168 & 0.106 \\
\hline Fatenin & period $x$ sex & & & 0.738 & 0.000 & 0.015 & 0.019 \\
\hline Proteas & $\mathrm{x}$ fatening peri & $1 \times$ sex & & 0.986 & 0.766 & 0.994 & 0.458 \\
\hline
\end{tabular}

BW- body weight at slaughter, ML - Metatarsus length, KL - keel length, BD - breast depth, TG thigh girth

${ }^{\mathrm{a}-\mathrm{e}}$ Means within a column with different superscripts differ significantly $(\mathrm{P}<0.05)$

In numerous studies, differences in the body weight of broiler chickens and the growth dynamics were also analyzed in terms of the effect of sex. Body weight was mostly significantly greater in males than in females (Ozkan et al. 1997, Bogosavljević-Bošković et al. 2006, 2011), whereas Pavlovski et al. 
(2007) determined better carcass conformation in males than in female chickens regardless of genotype and slaughter time i.e. chicken age, with no significant difference only for breast angle, similarly to the present research.

Based on absolute conformation measurements, relative conformation measurements were calculated, and the results are presented in Table 2.

The data in Table 2 indicate that male chickens, regardless of age (both at 7 and 9 weeks of age), had higher conformation indices compared to females $(\mathrm{P}<0.05)$. The two-week extension of the fattening period led to an increase in all conformation indices $(\mathrm{P}<0.05)$, given the increase in body weight, primarily through muscle growth in major carcass parts (breast, thighs, drumsticks). Also, interactions were most pronounced when induced by the fattening period and broiler sex, whereas carcass quality (expressed through conformation indices) was not affected by interactions among feed formulations, fattening periods and broiler sex (P>0.05). Similar results were obtained by Pavlovski et al. (2006) in their study on medium-growing RedBro chickens, which showed significantly better carcass conformation indices $(\mathrm{g} / \mathrm{mm})$ after the 8-day extension of the fattening period (from 42 to 50 days). As reported by Quentin et al. (2003), different rations primarily affect the growth of chickens, while carcass composition and some meat quality parameters remain practically unaffected. As the result of intensive broiler growth and greater body weights, chickens in the present experiment had greater relative conformation measurements compared to the findings of Blagojević et al. (2009) for the same hybrid under extensive farming and scantier feeding conditions for which the hybrid has been bred. Bogosavljević-Bošković et al. (2011) found that Hybro G males had statistically higher relative values for keel length and thigh girth $(\mathrm{P}<0.01)$ compared to female chickens.

\section{Conclusion}

The results of the present study revealed the following:

- intensive feeding of Master Gris chickens (relative to manufacturer's recommendations) led to greater final body weights of broilers and, hence, better carcass conformation traits, with very small differences due to feed formulation;

- two-week extension of the fattening period (from 49 to 63 days) resulted in better carcass conformation (all absolute and relative conformation measurements increased, with only breast angle value showing no significant difference $-\mathrm{P}>0.05$ );

- regardless of chicken age, females had better carcass conformation compared to females (sex had a significant effect on all absolute and relative carcass conformation measurements, except breast angle $\mathrm{P}>0.05)$. 


\section{Acknowledgment}

This study is part of Project Ref. No. 31033 titled "Sustainable Conventional and Revitalized Traditional Production of Value-Added Poultry Meat and Eggs", funded by the Ministry of Science and Technological Development of the Republic of Serbia.

\section{References}

Blagojević M. (2011): Uticaj genotipa na intenzitet porasta i kvalitet trupa i mesa brojlerskih pilića u ekstenzivnom sistemu gajenja. Doktorska disertacija. Univerzitet u Novom Sadu, Poljoprivredni fakultet, 1-116.

Blagojević M., Pavlovski Z., Škrbić Z., Lukić M., Milošević N., Perić L. (2009): The effect of genotype of broiler chickens on carcass quality in extensive rearing system. Acta veterinaria, 59 (1): 91-97.

Bogosavljevic-Boskovic S., Kurcubic V., Petrovic D.M., Radovic V. (2006): The effect of sex and rearing system on carcass composition and cut yields of broiler chickens. Czech Journal of Animal Science, 51 (1): 31-38.

Bogosavljević-Bošković S, Dosković V., Rakonjac S., Petrović D.M. (2011): Prinos i konformacija trupova brojlerskih pilića u zavisnosti od načina gajenja. International Scientific Symposium of Agriculture "Agrosym Jahorina 2011", 10-12.November 2011, Poljoprivredni fakultet, Istočno Sarajevo, RS, BiH - Proceedings: 296-301.

Commission Regulation (EC) No 543/2008. OJ L 157/46, 17.6.2008.

Dosković V., Bogosavljević-Bošković S., Lukić M., Škrbić Z., Rakonjac S., Petričević V. (2016): Effect of dietary protease supplementation and sex on dressing percentage and body conformation in broilers. Biotechnology in Animal Husbandry, 32 (2): 185193.

Hopić S., Pavlovski Z., Škrbić Z., Lukić M. (2002): Metode određivanja konformacije trupova pilića. Biotechnology in Animal Husbandry, 18 (3-4): 1-56.

Ozkan S., Settar P., Yalcin S. (1997): Effects of seasonal ambient temperature on yields of naked neck broilers ( $\mathrm{Na} / \mathrm{na}$ ) and their normally feathered (na/na) halfsibs. Poultry Meat Quality. In: Proc.XIII. Eur. Symp. Quality of Poultry Meat, Poznan, Poland. Session M-1: 49-52.

Pavlovski Zlatica, Lukić M., Cmiljanić R., Škrbić Zdenka (2006): Konformacija trupova pilića. Biotechnology in Animal Husbandry, 22 (3-4): 83-96.

Pavlovski Z., Škrbić Z., Cmiljanić R., Lukić M., Tomašević D. (2007): Uticaj sistema gajenja i bioloških faktora na konformaciju trupa i klanične osobine pilića u tovu. Biotechnology in Animal Husbandry, 23 (3-4): 59-66.

Quentin M., Bouvarel I., Berri C., Le Bihan-Duval E., Baéza E., Jégo Y., Picard M. (2003): Growth, carcass composition and meat quality response to dietary concentrations in fast-, medium-and slow-growing commercial broilers. Animal Research, 52 (1): 65-77.

Rakonjac S. (2016): Proizvodnja i kvalitet proizvoda kokoši nosilja iz alternativnih sistema gajenja. Doktorska disertacija, Univerzitet u Kragujevcu, Agronomski fakultet u Čačku. 
Reddish J.M., Lilburn M.S. (2004): A Comparison of Growth and Development Patterns in Diverse Genotypes of Broilers. 1. Male Broiler Growth. Poultry Science, 83 (7): 1067-1071.

Statsoft Inc. Statistica for Windows (2006): Version 5.0, Computer program manual. Tulsa: StatSoft Jnc.

Škrbić Z., Pavlovski Z., Lukić M., Milić D. (2011): The effect of rearing conditions on carcass slaughter quality of broilers from intensive production. African Journal of Biotechnology, 10 (10): 1945-1952.

Van Horne P.L.M., Van Harn J.J., Rodenburg T.B. (2004): Slow growing broilers: performance, mortality and welfare. XXI World's Poultry Congress, InstambulTurkey, 8-13 June 2004, Book of abstracts, p. 326. 


\title{
KONFORMACIJA TRUPOVA PILIĆA HIBRIDA MASTER GRIS KAO POKAZATELJ KVALITETA TRUPA
}

\author{
Vladimir Dosković ${ }^{1}$, Snežana Bogosavljević-Bošković ${ }^{1}$, Zdenka \\ $\breve{S}_{\text {Skbić }}^{2}$, Boban Jašović ${ }^{3}$, Simeon Rakonjac ${ }^{1}$, Veselin Petričević ${ }^{2}$, \\ Milun D. Petrović ${ }^{1}$ \\ ${ }^{1}$ Univerzitet u Kragujevcu, Agronomski fakultet u Čačku, , Cara Dušana 34, \\ 32000 Čačak \\ ${ }^{2}$ Istitut za stočarstvo, Autoput 16, P. Fah 23, 11080, Beograd-Zemun \\ ${ }^{3}$ Univerzitet u Prištini, Poljoprivredni fakultet Kosovska Mitrovica-Zubin potok- \\ Lešak, 38219 Lešak
}

\section{Rezime}

U radu je ispitivan uticaj različith formulacija hrane za tov pilića, vremena trajanja tova i pola pilića na apsolutne mere konformacije trupova (dužinu piska, dužinu kobilice, dubinu grudi, grudni ugao i obim bataka) i indekse mera konformacije trupova pilića medium-growing linijskog hibrida Master Gris. Na osnovu dobijenih rezultata, utvrđeno je da su pilići ovog hibrida, zahvaljujući intezivnom tovu, imali veću telesnu masu i bolju kvalitet trupa izražan preko mera konformacije trupa, nego što je to karakteristično za ovaj hibrid (jer je on namenjen za ekstenzivno gajenje). Petlići su, u odnosu na kokice, bez obzira na vreme klanja (49. ili 63.dan tova) imali bolju konformaciju trupa $(\mathrm{P}<0,05)$, a sa produžavanjem tova za 14.dana pilići oba pola su imali bolje i apsolutne i relativne mere konformacije trupa $(\mathrm{P}<0,05)$.

Ključne reči: pilići, konformacija trupa, hrana, uzrast pilića, pol. 